\title{
ROSA 2000 : les Recommandations pour le calcul aux états-limites des Ouvrages en Site Aquatique
}

\author{
Olivier Piet $^{1}$, Jean-Bernard Kovarik ${ }^{2}$, Manuel Le Moine ${ }^{3}$ \\ 'Ingénieur en Chef des Ponts et Chaussées, Centre d'Études \\ Techniques Maritimes Et Fluviales (CETMEF), Compiègne, France \\ Olivier.Piet@equipement.gouv.fr \\ 0344926015 \\ ${ }^{2}$ Ingénieur des Ponts et Chaussées, Port Autonome de Rouen, France \\ d2@rouen.port.fr \\ 0235525420 \\ ${ }^{3}$ Ingénieur des Travaux Publics de l'État, Centre d'Études \\ Techniques Maritimes Et Fluviales (CETMEF), Compiègne, France \\ Manuel.Le-Moine@equipement.gouv.fr \\ 0344926017
}

Résumé

Les Recommandations pour le calcul aux états-limites des Ouvrages en Site Aquatique, présentées sous la forme du cédérom ROSA 2000, ont été élaborées par le CETMEF grâce la collaboration de nombreux auteurs (ingénieurs, chercheurs, universitaires, praticiens avertis, etc.) ; elles servent de document d'application des Eurocodes aux projets d'ouvrages neufs maritimes et fluviaux, tout en harmonisant les règles de vérification selon le format semi-probabiliste aux états-limites. Le cédérom fournit au projeteur une aide hypertexte indispensable à la recherche et à la navigation dans cet ensemble très structuré de textes.

\section{Abstract}

The Guidelines for limit states design of maritime and waterways structures, presented in the form of the ROSA 2000 CD-ROM, have been developed by the CETMEF through the partnership of many authors (chief engineers, researchers, academics, skilled practitioners, etc.). These Guidelines assist the designer in applying Eurocodes to new projects in maritime and waterways works and in harmonizing design rules in coherence with limit states - semi probabilistic format. This CD-ROM provides the concept manager with hypertextual help making search and browsing easier within a structured set of texts.

\section{Le contexte}

Les Eurocodes ([3]), règles européennes de justification des constructions fondées sur le format semi-probabiliste aux états-limites, voient progressivement le jour et 
ne tarderont pas à s'imposer aux concepteurs de bâtiments et d'ouvrages de génie civil. Des principes analogues de vérification sont d'ailleurs déjà adoptés par les règlements français portant sur le béton armé ([14]), le béton précontraint ([12]) et les fondations ([13]).

Mais, en abordant le cas des ouvrages en site aquatique, le concepteur est confronté à des problèmes spécifiques :

- ces ouvrages sont moins bien connus que, par exemple, les bâtiments ou les ponts courants, et leur analyse est moins standardisée ;

- les actions ne sont en général pas décrites par des textes réglementaires mais sont laissées à la « libre » appréciation du concepteur, qui dispose pour cela de moins de lignes directrices;

- actuellement, les textes de référence officiels restent incomplets, notamment :

- des phénomènes importants ne sont pas couverts (par exemple, 1'action du sol sur les rideaux de soutènement);

- les textes sont obsolètes ou peu compatibles (par exemple, les règles de calcul des grues, se raccordent mal avec les règles de calcul des ouvrages de génie civil qui les supportent);

- certains textes procèdent d'une même doctrine (calcul aux étatslimites et approche semi-probabiliste de la sécurité) mais ne sont pas encore unifiés.

Le Ministère de l'Équipement, des Transports et du Logement (METL) a élaboré, par le biais du CETMEF, «ROSA $2000 »$ ([15]), recueil de recommandations dont l'objectif principal est de guider le concepteur pour l'application des Eurocodes aux projets d'ouvrages neufs maritimes et fluviaux.

\section{Qu'est-ce que le format semi-probabiliste?}

La méthode de calcul semi-probabiliste aux états-limites consiste à vérifier le dimensionnement d'un ouvrage de génie civil. Son application suppose donc que cet ouvrage est déjà pré-dimensionné.

Le projeteur doit commencer par recenser l'ensemble des situations de projet que l'ouvrage est susceptible de rencontrer au cours de sa vie. Une situation de projet peut être définie comme étant une étape de la vie de la structure déterminée par l'ensemble des conditions du projet régnant pendant un certain intervalle de temps durant lequel les paramètres intéressant la sécurité de l'ouvrage peuvent être considérés comme constants.

A chaque situation (classée en « durable», «transitoire », « accidentelle » voire «post-accidentelle ») correspond une liste des actions («permanentes » comme le poids des terres par exemple, « variables » comme les charges d'exploitation ou « accidentelles » comme le séisme), ou cas de charge, appliquées à la structure. 
Pour une situation de projet donnée, le concepteur doit vérifier le bon comportement de l'ouvrage vis-à-vis des états-limites pertinents. Un état-limite est un phénomène préjudiciable contre l'apparition duquel on souhaite prémunir l'ouvrage. Il existe deux classes principales d'états-limites :

- les états-limites de service (ELS), dont l'enjeu est modéré ; en général, ils correspondent à une perte d'aptitude au service de l'ouvrage ;

- les états-limites ultimes (ELU), dont l'enjeu est élevé ; en général, ils correspondent à une forme de rupture de l'ouvrage.

À chaque état-limite (glissement-plan, poinçonnement $\mathrm{du}$ sol de fondation, érosion régressive, résistance du béton armé, etc.), le projeteur associe une ou plusieurs combinaisons types d'actions. Comme l'indique Lequien ([9]), une combinaison type d'actions (rare, fréquente, quasi-permanente, fondamentale ou accidentelle) représente une sélection prédéfinie des valeurs représentatives des actions figurant dans le cas de charge considéré ; elle correspond théoriquement à une probabilité d'occurrence donnée au cours de la situation de projet étudiée.

On appelle « valeurs représentatives » les diverses valeurs défavorables possibles des grandeurs variables. Elles se distinguent généralement par leurs probabilités respectives d'occurrence ou de dépassement. On peut citer par exemple :

- la valeur caractéristique $X_{k}$; pour certains paramètres, elle correspond au fractile à $5 \%$ de sa loi de distribution supposée ;

- la valeur de calcul $X_{d}$; elle est souvent définie à partir de la valeur caractéristique par l'application d'un coefficient partiel $\left(X_{d}=X_{k} / \gamma_{M}\right)$.

La condition devant être remplie pour que l'état-limite ne soit pas atteint peut être traduite sous la forme d'une équation, appelée condition d'état-limite :

$$
R(X)-S(F) \geq 0
$$

[Équation 1]

où $R$ représente la résistance de la structure, $X$ les paramètres hors actions (matériaux, géométrie, interactions) qui la déterminent et $S$ l'effet des actions appliquées $F$. Les éléments moteurs $S$ sont également appelés sollicitations.

Le projeteur doit retenir les valeurs représentatives pertinentes de chaque paramètre, à partir desquelles il établit la condition de calcul à vérifier :

$$
R\left(\frac{X_{k}}{\gamma_{M}}\right)-S\left(\gamma_{f} \cdot F_{k}\right) \geq 0
$$

[Équation 2]

Dans cette nouvelle équation, où l'on compare les éléments de résistance $R$ aux sollicitations $S$ subies par l'ouvrage, les paramètres $X_{k}$ et $F_{k}$ sont les valeurs caractéristiques. Les coefficients $\gamma_{M}$ et $\gamma_{f}$ sont les coefficients partiels de sécurité. Les expressions $X_{d}=X_{k} / \gamma_{M}$ et $F_{d}=\gamma_{f} . F_{k}$ représentent les valeurs de calcul. 
Afin de tenir compte des incertitudes liées à la différence entre la modélisation théorique et la réalité du comportement, il convient d'introduire un coefficient de modèle. La condition de justification devient alors :

$$
\frac{1}{\gamma_{R d}} \times R\left(X_{d}\right)-\gamma_{S d} \times S\left(F_{d}\right) \geq 0 \quad \text { [Équation 3] }
$$

Si l'on note $R_{d}=R\left(X_{d}\right)$ et $S_{d}=S\left(F_{d}\right)$ les valeurs de calcul de la résistance et des sollicitations, l'équation 3 devient :

$$
\Gamma=\frac{R_{d} / \gamma_{R d}}{\gamma_{S d} \times S_{d}} \geq 1
$$

Le facteur de dimensionnement $\Gamma$ permet de qualifier la «position » d'un ouvrage (dont les dimensions sont supposées connues) vis-à-vis d'un état-limite donné. L'ouvrage est justifié si et seulement si $\Gamma \geq 1$ pour chaque état-limite.

$\mathrm{Au}$ final, on s'aperçoit que la vérification d'une structure selon le format semiprobabiliste réside dans la volonté de traiter, par le biais de coefficients partiels de sécurité, les incertitudes là où elles se trouvent. La démarche des justifications peut être résumée par l'organigramme suivant :

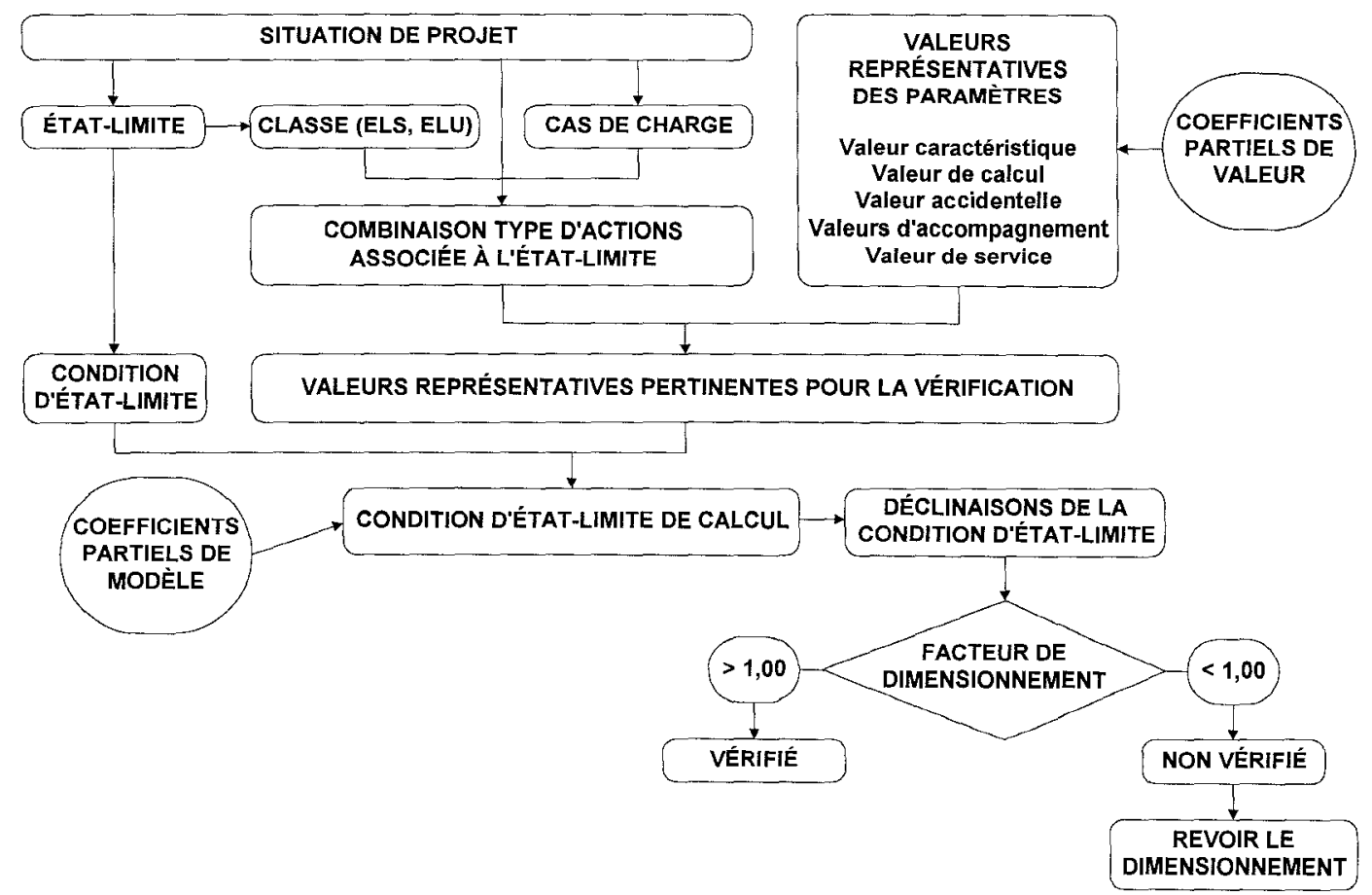

Figure 1: Organigramme des vérifications

Figure 1: The design flow chart 
Il convient de retenir que la méthode semi-probabiliste aux états-limites a pour objectif fondamental la maîtrise des risques, ce qui ne signifie pas leur suppression totale, mais leur écrêtement à un niveau jugé acceptable. Elle conduit à un dimensionnement optimisé des ouvrages pour un niveau de sécurité homogène et explicite; les matériaux seront donc bien répartis là où ils sont nécessaires, sans surdimensionnement.

Le format semi-probabiliste tire son nom de la méthode de détermination des coefficients partiels. Ceux-ci sont calculés par calibration probabiliste. Cette méthode est présentée dans les articles [1], [4] et [8].

Les coefficients partiels donnés dans ROSA 2000 ([15]) ont été déterminés, non pas par une calibration strictement probabiliste, mais :

- pour les coefficients de valeur $\left(\gamma_{\mathrm{g}}, \gamma_{\mathrm{q}}, \gamma_{\mathrm{M}}\right.$ ou $\left.\gamma_{\mathrm{R}}\right)$, à partir des règlements de calcul existants en France et à l'étranger ;

- pour les coefficients de modèle $\left(\gamma_{d}\right)$, par ajustement, en moyenne, sur les règles traditionnelles de vérification aux coefficients globaux, et pour certains d'entre eux par la traduction de règlements français existants.

\section{Que permet ROSA 2000?}

Les Recommandations pour le calcul aux états-limites des Ouvrages en Site Aquatique ([15]) ont été établies par un groupe de travail qui a coordonné les travaux rédactionnels entrepris par un grand nombre de professionnels représentatifs de multiples disciplines. Le champ couvert est celui des ouvrages maritimes « intérieurs » (quais-poids, gabions, appontements, ducs d'Albe, talus et pentes, rideaux de soutènement) et des ouvrages fluviaux (barrages, écluses, digues des voies navigables).

Les Recommandations sont orientées vers la prise en compte de la sécurité :

- elles précisent dans un format unifié l'ensemble des éléments nécessaires à la justification des ouvrages par le calcul ;

- elles indiquent les situations et les cas de charge les plus fréquemment rencontrés ;

- elles donnent les éléments méthodologiques indispensables à la détermination des valeurs représentatives des différents paramètres.

Le texte est structuré en cinq séries de fascicules :

- les dispositions communes, qui traitent pour l'essentiel du format de vérification et de différents paramètres hors actions (résistance, géométrie, matériaux, interaction);

- les équipements pour la prise en compte des actions qu'ils transmettent aux ouvrages (outillage, passerelles, défenses d'accostage, etc.); 
- les actions, qui donnent les lignes directrices pour la détermination des valeurs représentatives et des coefficients partiels à leur appliquer ;

o les ouvrages, qui présentent les états-limites pertinents, leurs modèles de calcul, les combinaisons types d'actions associées ;

- les documents d'application (guide pour faciliter l'utilisation des Recommandations dans les marchés de travaux, exemples commentés de notes de calcul, etc.).

Afin de faciliter la lecture des Recommandations, le CETMEF a demandé au Centre Scientifique et Technique du Bâtiment (CSTB) d'élaborer un logiciel convivial permettant :

- d'effectuer une recherche par mots-clés de chapitres pertinents, dans le prolongement de la Documentation des Techniques Routières Françaises (DTRF) ;

- de naviguer au sein du texte grâce à de nombreux liens hypertextes.

Ce logiciel a été baptisé ROSA 2000, par référence aux Recommandations.

Par exemple, si les mots-clés « Rideau de palplanches » et « Épaisseur des parties métalliques » sont choisis, on peut accéder à plusieurs articles, dont la partie traitant de la corrosion des palplanches du fascicule Rideaux de soutènement :

\subsubsection{CORROSION DES PALPLANCHES}

Lorsque la prise en compte de la Corrosion des rideaux do paiplanches siefectue par une surepaisseur sacrificielle, deux mêthodes peuvent etre mises en ceuvre (dans les situations corrodees):

- calcul des nouviles inerieas des palplanches avec les épaisseurs réduites, puis determination des efforts et verifications.

- ou détermination des efforts avec les rnerlies des palplanches non corrodées, puis vêffication de la résistance des sections d'épaisseur réduite.

Figure 2 : Résultat d'une recherche multicritères

Figure 2 : Result of a multicriterion research

A ce stade, on peut remarquer (voir la figure 2) la présence de deux types de liens hypertextes (cet article étant imprimé en noir et blanc, nous avons encadré les termes qui, à l'écran, apparaissent en couleur). Les liens pédagogiques, en bleu (ici, le mot «situations »), sont placés sur les termes propres au semi-probabilisme. Ils pointent vers la définition de ce mot dans le Glossaire. Les liens opérationnels, en vert (ici, les mots « corrosion » et « inerties »), sont placés quant à eux sur des termes "métier » et pointent vers d'autres articles des Recommandations. Par exemple, le lien placé sur le mot « corrosion » pointe vers le chapitre Épaisseur des parties métalliques du fascicule Paramètres géométriques. 
D'autres aides sont apportées à l'utilisateur, en sus du Glossaire : une présentation du semi-probabilisme par un diaporama avec des exemples très simples, une liste des symboles, une aide en ligne...

ROSA 2000 s'adresse aux acteurs des projets (maîtres d'ouvrages, maîtres d'œuvre, entreprises, bureaux d'études, etc.) et peut être commandé auprès des Presses de l'École Nationale des Ponts et Chaussées (télécopie : 01445827 44).

Une description détaillée de ROSA 2000 est disponible sur le site Internet du CETMEF ( http://www.cetmef.equipement.gouv.fr, rubrique «Les projets »). De plus amples renseignements peuvent être obtenus en écrivant à l'adresse :

Rosa2000.Cetmef@equipement.gouv.fr

\section{Conclusion}

Les Recommandations pour le calcul aux états-limites des Ouvrages en Site Aquatique ([15]) forment un ensemble cohérent qui exprime les règles de sécurité selon la méthode semi-probabiliste aux états-limites. Établies en conformité avec le format des Eurocodes ([3]), elles étendent les règles du BAEL ([14]), du BPEL ([12]) et du fascicule 62 Titre $V$ ([13]) aux projets d'ouvrages neufs en site aquatique.

L'un des avantages de ce format de vérification réside dans le fait qu'il permet d'identifier plus clairement les incertitudes tout en recherchant constamment l'homogénéité des approches pour les diverses parties de l'ouvrage.

Alors que les Eurocodes commencent déjà à s'imposer pour le calcul des ouvrages terrestres, le projeteur dispose aujourd'hui d'un outil pour appliquer ces règles européennes aux ouvrages maritimes et fluviaux.

On peut attendre de ces Recommandations, après une phase de familiarisation indispensable au cours de laquelle le retour d'expérience est attendu, d'améliorer la communication entre les acteurs du projet, de conduire à des ouvrages encore mieux optimisés et de favoriser l'évolution de la réglementation française en général vers les Eurocodes.

\section{Bibliographie}

[1] Besse, P., Berranger, I., Maroini, A. (1993) : « Méthodologie de calibration des codes semi-probabilistes ». Construction métallique $n^{\circ} 2-1993$. CTICM.

[2] Calgaro, J.-A. (1996) : «Introduction aux Eurocodes ». Presses de 1'école nationale des ponts et chaussées, 200 pages.

[3] Eurocodes. AFNOR. Voir aussi le site Internet des Euronormes relatives au génie civil : http://www.setra.fr/euronormes/Francais/PC Francais.htm 
[4] Goyet, J., Bureau, A. (1990): «Sécurité probabiliste des structures, évaluation des coefficients partiels de sécurité pour l'élaboration des nouveaux règlements ». Construction métallique $n^{\circ}$ 1-1990. CTICM.

[5] Goyet, J., Jacob, B., Lemaire, M., Mathieu, H. (1998) : « Dossier thématique - Fiabilité des constructions ». Revue française de génie civil, volume $2-n^{\circ} 5 / 1998$, pp. 527-610.

[6] Kovarik, J.-B. (1998) : «De l'application des Eurocodes aux ouvrages maritimes et fluviaux ». Revue française de génie civil, volume 2 $\mathrm{n}^{\circ}$ 5/1998, pp. 611-636.

[7] Kovarik, J.-B. (1999) : «La vérification des ouvrages maritimes aux étatslimites ». Bulletin de 1'AIPCN n ${ }^{\circ} 100$, pp. 35-39.

[8] Kovarik, J.-B. (2000) : «Qu'est-ce qu'un coefficient de sécurité en génie civil ». Revue française de génie civil, volume $4-n^{\circ}$ 6/2000, pp. 607-651.

[9] Lequien, P. (1993) : « Application de l'Eurocode 3 - Actions, combinaisons d'actions et applicabilité de l'EC3-DAN $»$. Construction métallique $n^{\circ} 1$ 1993. CTICM.

[10] Mathieu, H. et al. (2000) : «Risques et réglementation ». Risque et génie civil. Colloque des 8 et 9 novembre 2000 à Paris. Presses de l'école nationale des ponts et chaussées, pp. 41-55.

[11] Ministère de l'environnement et du cadre de vie (1979). «Instruction technique sur les directives communes de 1979 relatives au calcul des constructions ». Direction des journaux officiels.

[12] METL (1992) : «Règles techniques de conception et de calcul des ouvrages et constructions en béton précontraint suivant la méthode des états-limites ». BPEL 91. Fascicule $n^{\circ} 62$ - Titre I - Section II. D. des journaux officiels.

[13] METL (1993): «Règles techniques de conception et de calcul des fondations des ouvrages de génie civil ». Fascicule $n^{\circ} 62-$ Titre V. Direction des journaux officiels.

[14] METL (1999) : «Règles techniques de conception et de calcul des ouvrages et constructions en béton armé suivant la méthode des états-limites ». BAEL 91 révisé 99 . Fascicule $n^{\circ} 62$ - Titre I - Section I. D. des journaux officiels.

[15] METL (2001), Centre d'études techniques maritimes et fluviales (CETMEF) : "Recommandations pour le calcul aux états-limites des Ouvrages en Site Aquatique ». Cédérom ROSA 2000. Presses de l'école nationale des ponts et chaussées. Voir aussi le site Internet du CETMEF (rubrique «Les projets ») : http://www.cetmef.equipement.gouv.fr

[16] Piet, O. (2001) : «Le dimensionnement des ouvrages maritimes aux étatslimites ». Séminaire organisé par la Société hydrotechnique de France sur les «Conditions maritimes extrêmes : prévision, évolution et impacts sur la protection du littoral » le 5 juin 2001 à Paris.

[17] Revue française de géotechnique $n^{\circ} 93$ (4 ${ }^{\text {ème }}$ trimestre 2000), numéro consacré aux «Incertitudes en géotechnique et dimensionnement des ouvrages $\gg, 92$ pages. 